\title{
Modeling of Bit Error Rate in Cascaded 2R Regenerators
}

\author{
Öhman, Filip; Mørk, Jesper
}

Published in:

Journal of Lightwave Technology

Link to article, DOI:

10.1109/JLT.2005.862446

Publication date:

2006

Document Version

Publisher's PDF, also known as Version of record

Link back to DTU Orbit

Citation (APA):

Öhman, F., \& Mørk, J. (2006). Modeling of Bit Error Rate in Cascaded 2R Regenerators. Journal of Lightwave Technology, 24(2), 1057-1063. https://doi.org/10.1109/JLT.2005.862446

\section{General rights}

Copyright and moral rights for the publications made accessible in the public portal are retained by the authors and/or other copyright owners and it is a condition of accessing publications that users recognise and abide by the legal requirements associated with these rights.

- Users may download and print one copy of any publication from the public portal for the purpose of private study or research.

- You may not further distribute the material or use it for any profit-making activity or commercial gain

- You may freely distribute the URL identifying the publication in the public portal

If you believe that this document breaches copyright please contact us providing details, and we will remove access to the work immediately and investigate your claim. 


\title{
Modeling of Bit Error Rate in Cascaded 2R Regenerators
}

\author{
Filip Öhman and Jesper Mørk
}

\begin{abstract}
This paper presents a simple and efficient model for estimating the bit error rate (BER) in a cascade of optical reamplification and reshaping (2R) regenerators. The model includes the influences of amplifier noise, finite extinction ratio (ER), and nonlinear reshaping. The interplay between the different signal impairments and the regenerating nonlinearity is investigated. It is shown that an increase in nonlinearity can compensate for an increase in noise figure or decrease in signal power. Furthermore, the influence of the improvement in signal ER along the cascade and the importance of choosing the proper threshold of the nonlinearity are investigated.
\end{abstract}

Index Terms-Noise, optical communication, optical signal processing.

\section{INTRODUCTION}

$\mathbf{R}$ EGENERATION is one of the basic functionalities needed in an optical-communication network. Due to signal impairments from, e.g., attenuation, dispersion, noise, and crosstalk, the signal quality has to be restored at regular intervals in order to keep the accumulation of errors low. Alloptical reamplification, reshaping, and retiming (3R) regeneration has been demonstrated at $40 \mathrm{~Gb} / \mathrm{s}$ [1], [2] and over 100 regenerators have been cascaded [3], [4]. If the retiming is omitted, the remaining reamplification and reshaping is usually referred to as reamplification and reshaping $(2 \mathrm{R})$ regeneration. The signal reshaping can be implemented by the use of a device with a nonlinear thresholdlike transfer function, which improves the extinction ratio (ER) and reduces the variations of the one- and zero-levels due to, e.g., noise [5], [6]. This type of device can reduce the rate of error accumulation from amplifier noise and other impairments [7]-[9]; the stronger the nonlinearity, the slower the error accumulation. In practice, however, the realization of a stronger nonlinearity also results in more noise. For example, the cascading of two interferometers may increase the nonlinearity, but, at the same time, the increase of insertion loss requires further amplification, thus increasing the added spontaneous-emission noise. It is therefore important to understand the interplay between regenerator properties such as noise figure, nonlinearity, and extinction-ratio improvement. However, the analysis of the bit error rate (BER) in a cascade

Manuscript received June 22, 2005; revised September 19, 2005. This work was supported in part by the European network of excellence ePIXnet and the Danish research council through the Semiconductor Components for Optical Signal Processing (SCOOP) program.

The authors are with the Research Center COM, NanoDTU, Technical University of Denmark, DTU Bulid. 345v, DK-2800 Kgs. Lyngby, Denmark (e-mail: fo@com.dtu.dk; jm@com.dtu.dk).

Digital Object Identifier 10.1109/JLT.2005.862446 of many such devices is complicated by the need to take into account both random impairments and nonlinear redistribution processes. Reported theoretical investigations have either been very simplified [7] or computationally heavy [9]. Here, we expand the model of Mørk et al. [7], which investigated the interplay between noise and nonlinearity, to include a finite ER of the transfer function $\mathrm{ER}_{\mathrm{reg}}$, and of the signal entering the cascade $\mathrm{ER}_{\mathrm{in}}$, as well as the contribution to the mean power from the added amplified spontaneous emission (ASE) noise. The model allows us to investigate the interplay between noise, $\mathrm{ER}$, decision threshold, and nonlinearity in a $2 \mathrm{R}$ regenerator and is useful for analyzing and comparing different device types.

The model is presented in detail in Section II. In Section III, it is compared to the model in [9] and the basic assumptions are validated. Examples of results from the model are also presented and discussed. The final conclusions are drawn in Section IV.

\section{MODEL}

The building blocks of the model are shown in Fig. 1. The regenerator consists of an optical amplifier, a filter, a reshaping nonlinearity, and a variable attenuator. The variable attenuator is included in the regenerator model in order to keep the mean power level launched into the fibers constant between links, regardless of the added ASE and the nonlinear redistribution. The fiber links between the regenerators are considered as a loss, equal to the gain of the amplifier.

The basic assumption in the model is that the amplifier and reshaping nonlinearity are considered separately, as seen in Fig. 1. This picture obviously describes some physical implementations of the regenerator better than others. For example, it is a fairly accurate picture of a passive reshaping element, such as a fiber-based nonlinearity, with an external optical amplifier. For a semiconductor optical amplifier (SOA)-based regenerator, where the amplifying and reshaping element is the same physical medium, it is of course harder to conceptually separate the two functions. Furthermore, the reshaping nonlinearity is assumed to be described by a static piecewise linear transfer function, described in more detail in Section II-A, and the noise, which is described in Section II-B, is assumed to be Gaussian. Finally, the transformation of the signal, noise, and BER is described in detail in Section II-C.

\section{A. Transfer Function}

The nonlinear transfer function, i.e., the time-independent output power as a function of input power, is assumed to be 


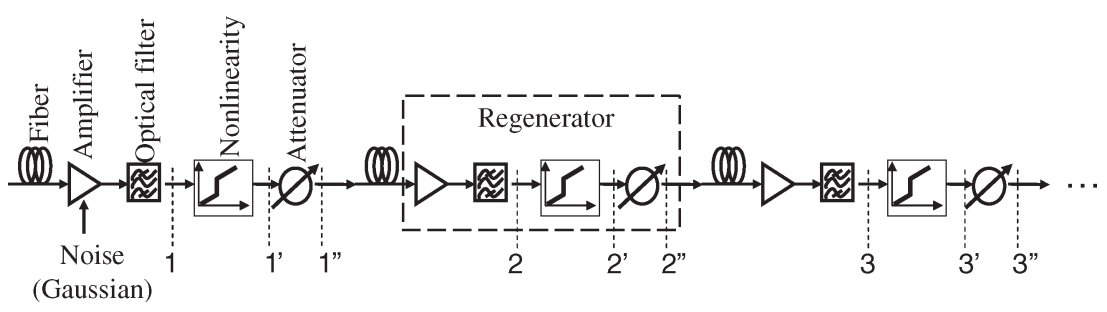

Fig. 1. Concept of the regenerator model and regenerator cascade.

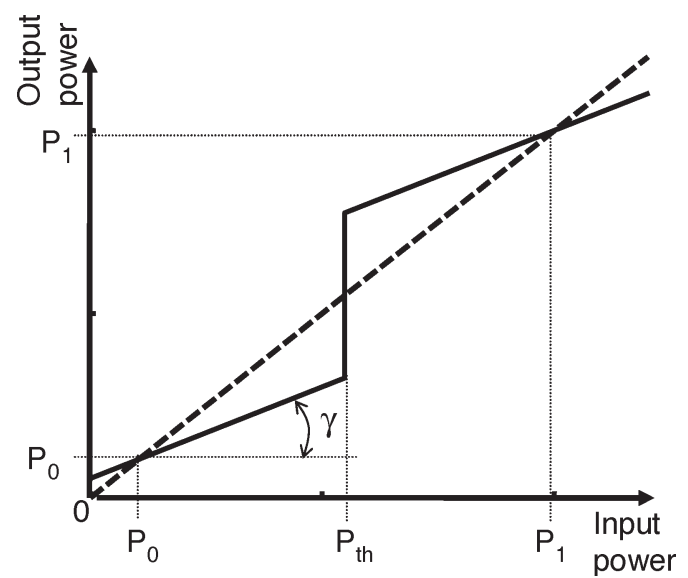

Fig. 2. Reshaping nonlinearity is described in the model by a piecewise linear transfer function.

a piecewise linear function, as seen in Fig. 2. This assumption is justified by the fact that nonlinearities with different shape but the same strength give very similar results for the BER in a cascade of regenerators [9].

The transfer function consists of two linear sections with slope $\gamma$ separated by an abrupt step at the threshold $\left(P_{\mathrm{th}}\right)$. There exist two stationary points of the function that are stable: one at the 1 level $\left(P_{1}\right)$ and one at the 0 level $\left(P_{0}\right)$. The finite $\mathrm{ER}$ of the regenerator transfer function, $\mathrm{ER}_{\mathrm{reg}}=P_{1} / P_{0}$, illustrates the fact that for some types of regenerators, e.g., wavelength converters, where the input signal is transferred to a new wavelength [10], even an infinite input ER might give a finite ER at the output.

The transfer function is described mathematically by

$$
\begin{array}{ll}
P_{\text {out }}=P_{0}+\gamma\left(P_{\text {in }}-P_{0}\right) ; & P_{\text {in }}<P_{\text {th }} \\
P_{\text {out }}=P_{1}+\gamma\left(P_{\text {in }}-P_{1}\right) ; & P_{\text {in }}>P_{\text {th }} .
\end{array}
$$

The slope of the linear parts controls the strength of the nonlinearity, $\gamma=1$ gives a linear function with the output power equal to the input power, and $\gamma=0$ gives a step function, with the two levels at $P_{0}$ and $P_{1}$ for the 0 and 1 levels, respectively. In this paper, the quantity $1-\gamma$ will be referred to as the strength of the nonlinearity, so that a low value, close to 0 , indicates a weak nonlinearity, and a high value, close to 1 , indicates a strong nonlinearity.

The use of a static time-independent transfer function implies that the bandwidth of the nonlinear response is assumed to be large in comparison to the detection bandwidth, and hence the bit rate of the system, so that dynamic effects, such as patterning and signal distortion from the regenerator, can be neglected. A more detailed analysis of patterning and bandwidth considerations has been made by Öhlén and Berglind [11], [12].

\section{B. Amplifier Noise}

The random intensity fluctuations of the signal at the logical 1 and 0 levels are described by probability density functions (PDFs). The contribution to the PDFs from the additional ASE noise added by the amplifier is considered to be Gaussian distributed with mean value $P_{\mathrm{ASE}}$ and standard deviation $\sigma_{\mathrm{ASE}}$. The addition of noise in the amplifier is described by a convolution of the input PDF with the PDF of the noise

$$
\mathrm{PDF}_{\text {out }}=\mathrm{PDF}_{\text {in }} \otimes \mathrm{PDF}_{\mathrm{ASE}}
$$

assuming that the ASE is independent of the input noise. The assumption of Gaussian noise is actually only a good approximation for not-too-small signal levels, where signalspontaneous beat noise dominates over, e.g., spontaneousspontaneous beat noise, which is the case for the 1 level. In this investigation, Gaussian noise will however be used for all signal levels, in order to simplify the calculations. It has been shown that a more rigorous model for the noise distribution gives similar BER as the Gaussian approximation [13] but with a different threshold. This means that the investigations of threshold dependence in this paper can only be considered qualitative. The amplifier in the model is considered as a linear amplifier with a gain that exactly compensates for the loss in the fiber links between regenerators. The linear amplifier allows the use of simple expressions for the ASE noise from the amplifier [14]. The contribution from ASE to the mean value of the signal is given by

$$
P_{\mathrm{ASE}}=F_{\mathrm{N}}(G-1) \hbar \omega_{0} B_{\mathrm{o}}
$$

where both polarizations are included, and $F_{\mathrm{N}}$ is the noise figure, assumed to be $2 n_{\mathrm{sp}}$, where $n_{\mathrm{sp}}$ is the spontaneous-emission factor. Furthermore, $G$ is the gain, $\hbar \omega_{0}$ is the photon energy, and $B_{\mathrm{o}}$ is the bandwidth of the optical filter. The contribution from the ASE to the width of the noise distribution is given by the expression for signal-spontaneous and spontaneousspontaneous beat noise [14]

$$
\begin{aligned}
\sigma_{\mathrm{ASE}, i, n}^{2}=2 F_{\mathrm{N}} & P_{\mathrm{sig}, i, n}(G-1) \hbar \omega_{0} B_{\mathrm{D}} \\
& +\left(\frac{F_{\mathrm{N}}}{2}(G-1) \hbar \omega_{0}\right)^{2} B_{\mathrm{D}}\left(2 B_{\mathrm{o}}-B_{\mathrm{D}}\right)
\end{aligned}
$$

where $\sigma_{\mathrm{ASE}}^{2}$ is the variance of the beat noise, $P_{\mathrm{sig}, i, n}$ is the signal power of the 1 or 0 level at regenerator number $n$, 
and $B_{\mathrm{D}}$ is the effective device bandwidth of the reshaping element, which is assumed to be smaller than the bandwidth of the optical filter. The relatively small effective bandwidth that is used in this paper is motivated by the limited bandwidth of typical semiconductor devices, where the response time is limited by slow carrier relaxations. For faster nonlinearities, the effective bandwidth of the nonlinearity might very well be larger than for the optical filter, which then becomes the main limiting factor for the noise in (4).

The use of beat noise as a measure of the noise added in the amplifiers is motivated by the description of the reshaping element in terms of a transfer function. It has been argued [8] that this intensity transformation corresponds to detection as far as conversion from ASE noise to intensity variations is concerned.

\section{Signal, Noise, and BER Transformations}

The convolution expressed by (2) gives an output noise variance from the amplifier, which is the sum of the input and internal noise variances

$$
\sigma_{\text {out }, \mathrm{amp}}^{2}=\sigma_{\mathrm{in}, \mathrm{amp}}^{2}+\sigma_{\mathrm{ASE}}^{2}
$$

and a mean power that is

$$
P_{\mathrm{out}, \mathrm{amp}}=P_{\mathrm{in}, \mathrm{amp}} G+P_{\mathrm{ASE}} .
$$

The linear transformation used in the regenerators changes the standard deviation by a factor equal to the slope $\gamma$, and the attenuator just gives a constant factor $L_{\text {att }}$ representing a linear loss

$$
\sigma_{\mathrm{out}}=L_{\mathrm{att}} \gamma \sigma_{\mathrm{in}}
$$

Assuming a noiseless signal at the input to the cascade and with the numbering of Fig. 1, the evolution of the variance of the signal in the cascade can thus be described as

$$
\begin{aligned}
\sigma_{i, 1}^{2}= & \sigma_{\mathrm{ASE}, i, 1}^{2} \\
\sigma_{i, 1^{\prime \prime}}^{2}= & L_{\mathrm{att}} \gamma \sigma_{i, 1}^{2} \\
\sigma_{i, 2}^{2}= & \sigma_{i, 1^{\prime \prime}}^{2}+\sigma_{\mathrm{ASE}, i, 2}^{2}=L_{\mathrm{att}} \gamma \sigma_{i, 1}^{2}+\sigma_{\mathrm{ASE}, i, 2}^{2} \\
& \cdots \\
\sigma_{i, n}^{2}= & L_{\mathrm{att}} \gamma \sigma_{i, n-1}^{2}+\sigma_{\mathrm{ASE}, i, n}^{2} \\
= & \sum_{k=1}^{n}\left(L_{\mathrm{att}} \gamma\right)^{2(n-k)} \sigma_{\mathrm{ASE}, i, k}^{2} .
\end{aligned}
$$

As long as equal slopes of the transfer function are assumed for both the 1 and 0 levels, (8) is valid for both.

The mean value of the signal levels and ASE will evolve in the cascade as

$$
\begin{aligned}
& P_{i, n}=\left(L_{\mathrm{att}} \gamma\right)^{n-1} P_{i, s}+\frac{\left(L_{\mathrm{att}} \gamma\right)^{n-1}-1}{L_{\mathrm{att}} \gamma-1} L_{\mathrm{att}}(1-\gamma) P_{i} \\
& +\frac{\left(L_{\mathrm{att}} \gamma\right)^{n}-1}{L_{\mathrm{att}} \gamma-1} P_{\mathrm{ASE}}
\end{aligned}
$$

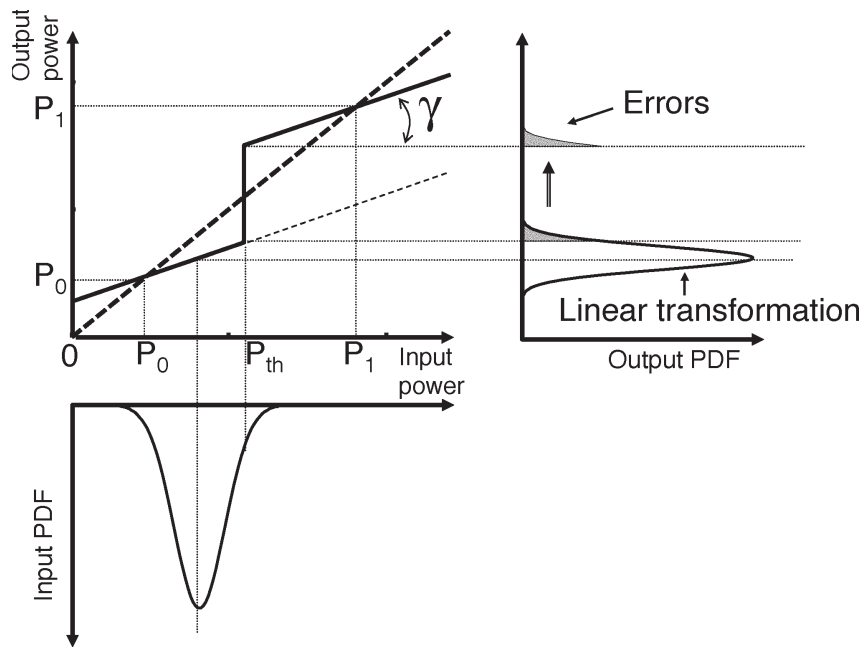

Fig. 3. Nonlinear transfer function and the concept of a piecewise linear transformation of the PDF.

where $i=0,1$ represent the 0 and 1 levels, respectively, $P_{i, s}$ are the mean powers of the respective levels at the start of the cascade, and $P_{i}$ are the stable points of the transfer function shown in Fig. 2. It is assumed that the threshold is situated in between the power levels of the two logical symbols at the start of the cascade.

The PDFs of the 1 and 0 levels at the output of the nonlinearity are assumed to be described by the transformation of the input PDFs using the linear parts of the transfer function, extended beyond the threshold, plus a part describing the unrecoverable errors at that regenerator, as shown in Fig. 3 for the 0 level. The latter part will constitute the increase in BER at the particular regenerator considered. A more correct transformation of the PDF using the nonlinear transfer function would give a non-Gaussian distribution [6], [9] at the output of the regenerator. The PDF can then no longer be described using only the mean value and variance but has to be described in detail through the cascade of regenerators, including the convolution with the amplifier noise and the nonlinear transformation. The semilinear approximation used here does, however, greatly simplify the calculations. Furthermore, this assumption includes the part of the PDFs above (or below for the 1 level) the threshold twice, both as errors at one regenerator and also linearly transformed to the output and sent further to the next regenerator, where it potentially contributes to even more errors. This additional error is largest for a linear transfer function and 0 for an ideal step function. As will be shown later, it is an excellent approximation for not-too-large BER values.

The errors, for $0 \mathrm{~s}$ and $1 \mathrm{~s}$, respectively, added in amplifier number $n$ can be described, using the Gaussian assumption [15], as

$$
\begin{aligned}
E\left(\sigma_{i, n}, P_{i, n}\right) & =\frac{1}{2} \operatorname{erfc}\left(\frac{\left|P_{\mathrm{th}}-P_{i, n}\right|}{\sqrt{2} \sigma_{i, n}}\right) \\
& \approx \frac{1}{\sqrt{2 \pi}} \frac{\sigma_{i, n}}{\left|P_{\mathrm{th}}-P_{i, n}\right|} \exp \left(-\frac{\left(P_{\mathrm{th}}-P_{i, n}\right)^{2}}{2 \sigma_{i, n}^{2}}\right)
\end{aligned}
$$

where $\operatorname{erfc}(\bullet)$ is the complementary error function. 
The evolution of the BER can be described by noticing that the reshaping elements do not add any errors, but just collects the errors added by the noise in the amplifier, and that the attenuator is just a linear loss, which does not change the BER. Furthermore, we analyze the BER for the 1 and 0 levels separately so that

$$
\mathrm{BER}=\frac{1}{2}\left(\mathrm{BER}_{0}+\mathrm{BER}_{1}\right)
$$

where the 1 and 0 bits are assumed to appear with equal probability. The result, which is the same for both 1's and 0 's, is

$$
\begin{aligned}
\operatorname{BER}_{i, 1}= & E\left(\sigma_{i, 1}, P_{i, 1}\right) \\
\operatorname{BER}_{i, 1^{\prime \prime}}= & \operatorname{BER}_{i, 1} \\
\operatorname{BER}_{i, 2}= & \operatorname{BER}_{i, 1}+E\left(\sigma_{i, 2}, P_{i, 2}\right) \\
\operatorname{BER}_{i, 2^{\prime \prime}}= & \operatorname{BER}_{i, 2} \\
& \cdots \\
\operatorname{BER}_{i, n}= & \operatorname{BER}_{i, n-1}+E\left(\sigma_{i, n}, P_{i, n}\right)
\end{aligned}
$$

where the numbering refers to Fig. 1. The BER is thus completely described by the evolution of the mean values and standard deviations for the 1 and 0 levels of the signal.

Now, finally, the BER for the 0 and 1 levels after $N$ regenerators can be expressed by the use of (8), (9), and (12) as

$$
\mathrm{BER}_{i, N}=\frac{1}{\sqrt{2 \pi}} \sum_{n=1}^{N} \frac{\sigma_{i, n}}{\left|P_{\mathrm{th}}-P_{i, n}\right|} \exp \left(-\frac{\left(P_{\mathrm{th}}-P_{i, n}\right)^{2}}{2 \sigma_{i, n}^{2}}\right) .
$$

The sum in (13) cannot, as far as the authors know, be evaluated analytically. In [7], it was shown that for the special case of infinite ER, both for the transfer function and the input signal, and where the contribution to the mean power by the ASE is neglected, it is possible to write down a good analytical approximation to the sum. In that approximation, the noise variance $\sigma_{\mathrm{ASE}}^{2}$ is calculated for the 1 level, neglecting the spontaneous-spontaneous beat noise, and the same variance is used also for the 0 level. The result is [7]

$$
\mathrm{BER}_{N} \cong \frac{1}{\sqrt{\frac{2 \pi Q_{0}^{2}}{F_{\mathrm{N}}}}} N \sqrt{\frac{1-\gamma^{2}}{1-\gamma^{2 N}}} \exp \left(-\frac{1}{2} \frac{Q_{0}^{2}}{F_{\mathrm{N}}} \frac{1-\gamma^{2}}{1-\gamma^{2 N}}\right)
$$

where the normalized mean input power is

$$
Q_{0}^{2}=\frac{\bar{P}_{\text {in }}}{4 \hbar \omega_{0} B_{e}} .
$$

Although an analytical expression gives more direct insight into the interplay between nonlinearity and noise, the more general expression in (13) is still very useful as it is simple and efficient to evaluate numerically. It also allows us to investigate the influence of ER improvements in a cascade of regenerators.

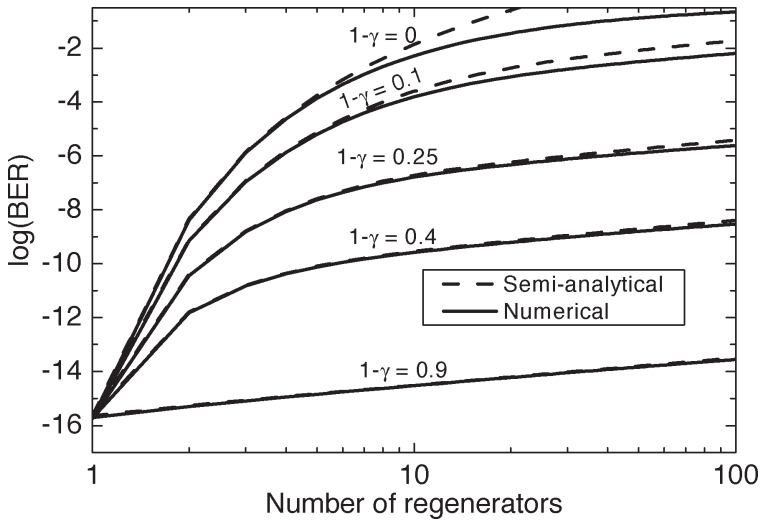

Fig. 4. Comparison of BER as a function of number of cascaded regenerators calculated with the semianalytical model presented in this paper and the numerical model in [9].

\section{RESULTS AND DISCUSSION}

\section{A. Model Validation}

First, the piecewise linear semianalytical model of this paper is compared to the numerical calculation of the BER in a cascade of regenerators from [9] in Fig. 4. In the comparison, the influence of the ASE mean power has been neglected since this is not included in the numerical model. The noise figure was $8 \mathrm{~dB}$, the device bandwidth $40 \mathrm{GHz}$, the mean power at the input of the cascade was $-3 \mathrm{dBm}$, the gain was $20 \mathrm{~dB}$, the input $\mathrm{ER}, \mathrm{ER}_{\mathrm{in}}=10 \mathrm{~dB}$, and the regenerator had an $\mathrm{ER}_{\text {reg }}$ of $10 \mathrm{~dB}$.

As seen in the figure, the two models give similar results, except for the linear case. The reason for the obviously erroneous result of the semianalytical model $($ BER $>1)$ in this case is the extra errors introduced by assuming that the PDF at the output consists of a linear transformation of the input PDF plus a part that represents the unrecoverable errors. In principle, this adds extra errors also for stronger nonlinearities and only for the ideal step function does this approximation not give additional errors. However, as the calculations show, the approximation is already a good one for fairly modest nonlinearities or for small BER values.

Fig. 4 further shows the regenerating capability of the nonlinear intensity transformation and the effect of a stronger nonlinearity. A single regenerator can never improve the BER, which in this case can be seen by noting that all regenerators give the same BER after one link, regardless of the strength of the nonlinearity. The effect of the regeneration is to reduce the rate at which errors accumulate in a cascade of regenerators; a stronger nonlinearity gives a slower increase of errors in the cascade. However, it should be noted that errors from timing jitter have been neglected in this analysis. A stronger nonlinearity can increase timing jitter if retiming is not used [16], which means that in $2 \mathrm{R}$ regeneration, there is a tradeoff between intensity fluctuations and timing jitter.

\section{B. Mechanisms of Regeneration}

The use of a regenerator to suppress the mean power in the 0 bits while leaving the 1 bits unchanged, and hence increase the ER of the signal, is probably the most straightforward 


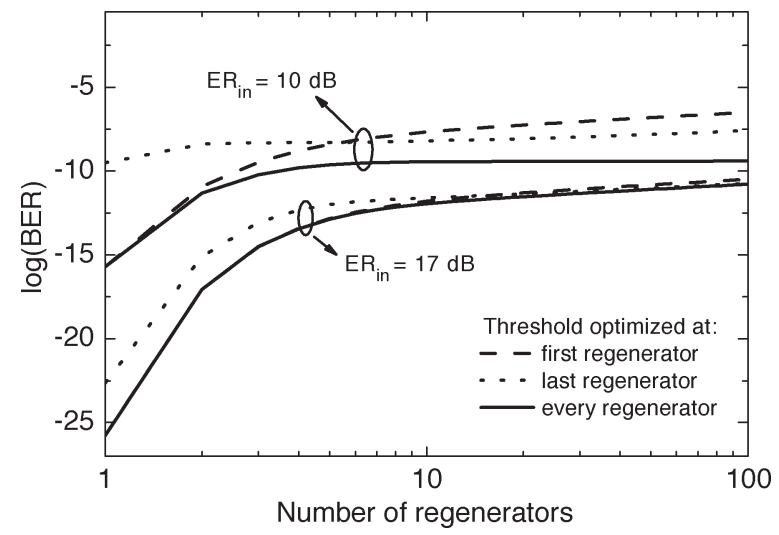

Fig. 5. BER as a function of number of regenerators for three choices of threshold, optimum (solid line), fixed and adjusted to the input signal (dashed line), and a fixed threshold optimized for low BER at the last regenerator (dotted line). The other parameter values were: mean input power into the cascade $P_{\text {in }}=-3 \mathrm{dBm}, 1-\gamma=0.25, B_{\mathrm{D}}=40 \mathrm{GHz}, B_{\mathrm{o}}=125 \mathrm{GHz}$, $F_{\mathrm{N}}=8 \mathrm{dBm}, G=20 \mathrm{~dB}$, and $\mathrm{ER}_{\text {reg }}=20 \mathrm{~dB}$. For the upper set of lines, the input ER was $10 \mathrm{~dB}$ and for the lower set $17 \mathrm{~dB}$.

application of a regenerator. In order to properly use the improvement of the ER, it is important to choose the proper threshold of the regenerator. This is shown in Fig. 5, where the BER, as a function of the number of regenerators in the cascade, is plotted for three different choices of threshold and two different input ERs.

The solid lines show the result when the threshold is optimized for each regenerator to give as few errors as possible at that regenerator. For the dashed lines, the threshold was kept constant for all regenerators at the optimum value for the first regenerator of the cascade. Finally, for the dotted lines, a fixed threshold for all regenerators was also used, but the threshold value was optimized for minimum BER at the last regenerator in the cascade.

Obviously, the best result is always achieved when the threshold is optimized for each regenerator. If the optimization is not possible for practical reasons (the simultaneous optimization of several tens or even hundreds of regenerators seems like a daunting task) and one fixed threshold needs to be used, Fig. 5 gives some indications on the considerations that have to be made. For a large input ER, in this case $17 \mathrm{~dB}$, the ER improvement in the cascade is not that important and only a small difference is seen between the two choices. For a lower input ER, $10 \mathrm{~dB}$ in this case, the changes through the cascade can be substantial and the choice of threshold has to be made in relation to how many regenerators are cascaded. For a few regenerators, the ER has not changed appreciably from the input to the cascade, and it is advantageous to optimize the threshold at the first regenerator. For many regenerators, where the signal has been strongly affected by the nonlinear transfer functions, the ER has been improved and the optimization should be made at the last regenerator.

In Figs. 6, 8, and 9, the results are presented in a way to show the interplay between parameters. Instead of explicitly showing the BER as a function of the number of regenerators, the relationship between two regenerator parameters is investigated at a specific BER and number of regenerators. The lines in the figure are thus contour lines that describe the relation between

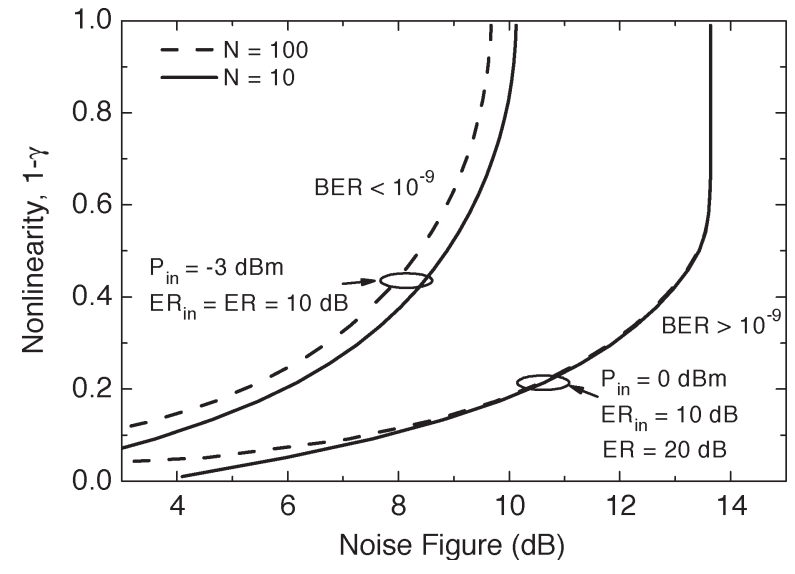

Fig. 6. Nonlinearity as function of noise figure for BER $=10^{-9}$ and different number of regenerators at mean input power of -3 and $0 \mathrm{dBm}$. The input ER was $10 \mathrm{~dB}$, and the transfer-function ER was $20 \mathrm{~dB}$ in the $0-\mathrm{dBm}$ case and 10 in the $-3-\mathrm{dBm}$ case. The other parameters were $B_{\mathrm{D}}=40 \mathrm{GHz}$, $B_{\mathrm{O}}=125 \mathrm{GHz}$, and $G=20 \mathrm{~dB}$.

the two parameters that give a BER of $10^{-9}$. The lines divide the parameter space into regions where the BER is larger or smaller than $10^{-9}$. In this way, it is possible to examine the interplay between these two parameters. The threshold values in these figures were always optimized for every regenerator.

In Fig. 6, the interplay between the nonlinearity and noise figure is investigated. The nonlinearity is plotted as a function of noise figure for different number of regenerators. Two different cases are plotted: one with $-3 \mathrm{dBm}$ mean input power into the cascade and one with $0 \mathrm{dBm}$. The regenerator ER was 10 and $20 \mathrm{~dB}$, respectively. The input ER was $10 \mathrm{~dB}$ in both cases.

The ability of a stronger nonlinearity to compensate for an increase in noise figure can clearly be seen. For example, a 3-dB increase in noise figure around a noise figure of $6 \mathrm{~dB}$ in the 0-dBm-input-power case can be compensated by a modest decrease of $\gamma$ of about $10 \%$. Put in another way, this means that it might be beneficial to increase the nonlinearity at the expense of a higher noise figure, by, e.g., the cascading of several regenerators in the same node [17]. The benefit of increasing the nonlinearity is also larger for a larger number of regenerators, as seen when comparing the curves for 10 and 100 regenerators for low noise figures. In order to get an idea of how the reshaping elements affect the noise figure, we consider it as a linear attenuator, which has to be compensated for by additional gain, and study the simple case of a cascade of linear gain and loss media. The effective noise figure of the attenuating elements is then equal to the loss, and the total noise figure can be expressed as [18]

$$
F_{\mathrm{Ntot}}=F_{\mathrm{N} 1}+\frac{F_{\mathrm{N} 3}}{G_{1} L_{2}}+\frac{F_{\mathrm{N} 5}}{G_{1} L_{2} G_{3} L_{4}}+\cdots
$$

where $F_{\mathrm{N}}$ is the noise figure, $G$ is the gain, and $L$ is the loss. The numbering refers to the order of the elements in the cascade, where the gain media have odd numbers, and the loss media have even numbers. From (16), it is clear that the total noise figure of the cascade is minimized by having a high gain in the first amplifier and as low losses as possible. In the case 


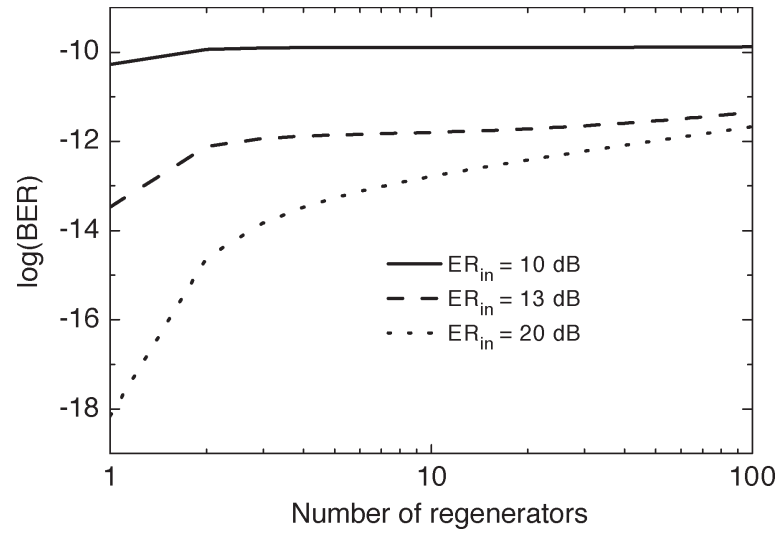

Fig. 7. BER as function of the number of cascaded regenerators for different input ERs. The ER of the regenerator was $20 \mathrm{~dB}$ in all cases, and the other parameters were $1-\gamma=0.5, F_{\mathrm{N}}=10 \mathrm{~dB}, P_{\text {in }}=-3 \mathrm{dBm}, B_{\mathrm{D}}=40 \mathrm{GHz}$, $B_{\mathrm{o}}=125 \mathrm{GHz}$, and $G=20 \mathrm{~dB}$.

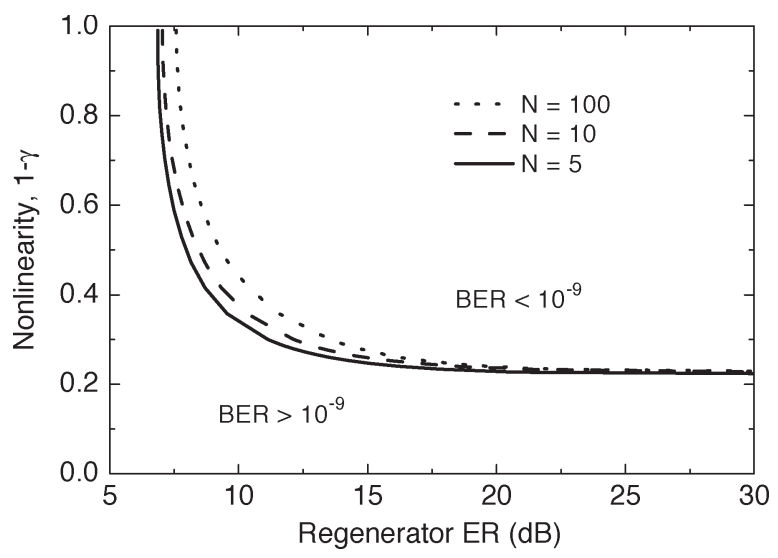

Fig. 8. Nonlinearity as a function of regenerator ER. The ER of the input signal was $10 \mathrm{~dB}$ in all cases, and the other parameters were $F_{\mathrm{N}}=8 \mathrm{~dB}$, $P_{\text {in }}=-3 \mathrm{dBm}, B_{\mathrm{D}}=40 \mathrm{GHz}, B_{\mathrm{o}}=125 \mathrm{GHz}$, and $G=20 \mathrm{~dB}$.

of a nonlinear reshaping element, the loss should be high for the 0 level, while the 1 level should experience as low loss as possible. For active SOA-based regenerators, the reshaping element can actually have gain, but the tradeoff in that case is the relatively high noise figure of SOAs (about $8 \mathrm{~dB}$ ) compared to EDFAs (about $5 \mathrm{~dB}$ ). In Fig. 6, this should be compared to a typical value for the nonlinearity. An SOA Mach-Zehnder interferometer has a nonlinearity $(1-\gamma)$ of about 0.35 [9], which allows for about 8-dB additional noise figure compared to the linear case for ten regenerators, $0 \mathrm{dBm}$ input power, and the operating conditions given in Fig. 6. This kind of investigation can be used to compare the cascading properties of different devices and regenerator types with known nonlinearity and noise figure. Looking at the other end of the curve, where the nonlinearity is close to an ideal step function, $\gamma$ plays a smaller role. When the BER is dominated by the errors added in the first few regenerators, due to a high noise figure, a further increase in nonlinearity is not beneficial.

In the $-3-\mathrm{dBm}$ case, the input and regenerator ER is the same, as opposed to the $0-\mathrm{dBm}$ case. The small difference between the results for 10 and 100 regenerators in the $0-\mathrm{dBm}$ case shows the importance of the ER improvement. The improvement makes the errors added later in the cascade very

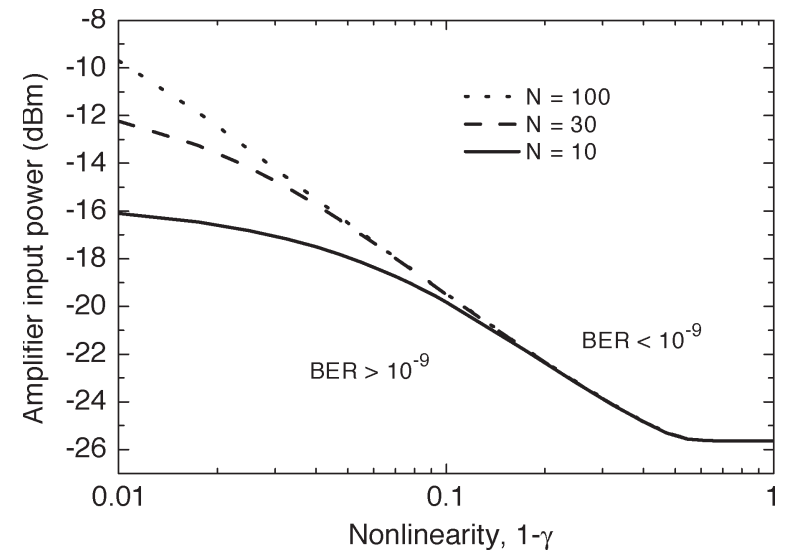

Fig. 9. Amplifier input power as a function of nonlinearity. The parameters were $B_{\mathrm{D}}=40 \mathrm{GHz}, B_{\mathrm{O}}=125 \mathrm{GHz}, F_{\mathrm{N}}=8 \mathrm{~dB}, G=20 \mathrm{~dB}, \mathrm{ER}_{\mathrm{reg}}=$ $20 \mathrm{~dB}$, and $\mathrm{ER}_{\mathrm{in}}=10 \mathrm{~dB}$.

small compared to the errors added in the first few regenerators. This is shown in more detail in Fig. 7, where the BER is plotted as a function of the number of cascaded regenerators for different values of the input ER.

For the case where the input ER is low, the BER is completely dominated by the errors from the first two regenerators where the ER is lowest. As the ER is improved, the rate of error accumulation decreases. It is not until the accumulated errors from a large number of regenerators with high input ER becomes comparable to the errors from the first regenerators that the BER increases appreciable again, as seen in the 13-dB case in Fig. 7. This effect is also seen in Fig. 8, where the nonlinearity needed for a BER of $10^{-9}$ is plotted as a function of regenerator ER at an input ER of $10 \mathrm{~dB}$. For a large regenerator ER, the signal is strongly improved in the beginning of the cascade, and the number of errors added in later regenerators is relatively small. This is seen by noting that the lines for a different number of regenerators coincide for a large ER.

The main information from Fig. 8, however, regards the interplay between regenerator nonlinearity and ER. For a large ER, a small increase in nonlinearity can improve the cascadability substantially or compensate for quite a large decrease in ER. This result agrees qualitatively with the findings by Hainberger et al. [8]. The reason for this result is that at high $\mathrm{ER}$, the error-accumulation rate is mainly given by the beat noise at the 1 and 0 levels, i.e., the widths of the PDFs. The only way to reduce these is to redistribute the noise using a stronger nonlinearity. Fig. 8 also shows the importance of achieving a good ER in regenerators, since a low ER strongly impairs the performance.

In Fig. 9, the input power needed to achieve a BER of $10^{-9}$ is plotted as a function of the nonlinearity for a different number of regenerators at a noise figure of $8 \mathrm{~dB}$. The gain and the link loss was $20 \mathrm{~dB}$, which means that the mean input power into the cascade is $20 \mathrm{~dB}$ higher than into the amplifier.

Again, it is seen that a small increase in nonlinearity can compensate for signal degradation, especially for a large number of regenerators. In this case, a stronger nonlinearity can increase the distance a signal can be transmitted between 
regenerators or decrease the transmission power needed in the system by improving the power budget.

\section{CONCLUSION}

In this paper, $2 \mathrm{R}$ regeneration in an optical-communication system was investigated theoretically for the case where the regenerator can be described by a nonlinear intensity transfer function. By considering the evolution of the PDFs of 0 and 1 levels, a simple and efficient model for the evolution of the BER in a cascade of regenerators was developed. The model takes into account the evolution of noise, mean power level, and ER along the cascade and the efficiency of the model makes it possible to investigate in detail the interplay between parameters characterizing the regenerator. The model showed that in order to utilize the increase in ER in a cascade of regenerators the threshold value of the nonlinear transfer function has to be optimized properly and the optimal threshold value depends on the number of cascaded regenerators. Finally, the interplay between the amount of reshaping through the nonlinearity and the other regenerator parameters was investigated. It was shown that a fairly small increase in nonlinearity, in many cases, can compensate for a degradation of noise figure or input power. These results should be useful in analyzing and comparing different regenerator implementations and optimizing the designs.

\section{REFERENCES}

[1] O. Leclerc et al., "Optical regeneration at $40 \mathrm{~Gb} / \mathrm{s}$ and beyond," J. Lightw. Technol., vol. 21, no. 11, pp. 2779-2790, Nov. 2003.

[2] C. Bornholdt, J. Slovak, and B. Sartorius, "Semiconductor-based alloptical 3R regenerator demonstrated at $40 \mathrm{~Gb} / \mathrm{s}$," Electron. Lett., vol. 40, no. 3, pp. 192-193, Feb. 2004.

[3] B. Lavigne, P. Guerber, P. Brindel, E. Balmefrezol, and B. Dagens, "Cascade of 100 optical 3R regenerators at $40 \mathrm{~Gb} / \mathrm{s}$ based on all-active Mach-Zehnder interferometers," in Proc. Eur. Conf. Optical Communication (ECOC), Amsterdam, The Netherlands, 2001, p. 290.

[4] J. Leuthold, J. Jaques, and S. Cabot, "All-optical wavelength conversion and regeneration," in Proc. Optical Fiber Communication (OFC), Los Angeles, CA, 2004, p. 803.

[5] L. J. Christiansen, L. Xu, K. Yvind, F. Öhman, L. Oxenløve, and J. Mørk, " $2 \mathrm{R}$ regeneration in concatenated semiconductor optical amplifier and electroabsorbers," in Proc. Eur. Conf. Optical Communication (ECOC), Stockholm, Sweden, 2004, pp. 30-31, Paper Mo3.4.3.

[6] F. Öhman, S. Bischoff, B. Tromborg, and J. Mørk, "Noise and regeneration in semiconductor waveguides with saturable gain and absorption," IEEE J. Quantum Electron., vol. 40, no. 3, pp. 245-255, Mar. 2004.

[7] J. Mørk, F. Öhman, and S. Bishoff, "Analytical expression for the bit error rate of cascaded all-optical regenerators," IEEE Photon. Technol. Lett., vol. 15 , no. 10, pp. 1479-1481, Oct. 2003.
[8] R. Hainberger, T. Hoshida, S. Watanabe, and H. Onaka, "BER estimation in optical fiber transmission systems employing all-optical 2R regenerators," J. Lightw. Technol., vol. 22, no. 3, pp. 746-754, Mar. 2004.

[9] P. Öhlen and E. Berglind, "Noise accumulation and BER estimates in concatenated nonlinear optoelectronic repeaters," IEEE Photon. Technol. Lett., vol. 9, no. 7, pp. 1011-1013, Jul. 1997.

[10] K. Obermann and K. Petermann, "Estimation of BER performance and cascadability of wavelength converters based on cross-gain modulation in semiconductor optical amplifiers," Proc. Inst. Elect. Eng.—Optoelectron., vol. 147, no. 2, pp. 133-137, Apr. 2000.

[11] P. Öhlen and E. Berglind, "Measurements and modelling of patterndependent BER and jitter in reshaping optoelectronic repeaters," Proc. Inst. Elect. Eng.—Optoelectron., vol. 147, no. 2, pp. 97-103, Apr. 2000.

[12] — - "BER caused by jitter and amplitude noise in limiting optoelectronic repeaters with excess bandwidth," Proc. Inst. Elect. Eng.Optoelectron., vol. 145, no. 3, pp. 147-150, Jun. 1998.

[13] G. H. Einarsson, Principles of Lightwave Communication, 1st ed. New York: Wiley, 1996.

[14] N. A. Olsson, "Lightwave systems with optical amplifiers," J. Lightw. Technol., vol. 7, no. 7, pp. 1071-1082, Jul. 1989.

[15] G. P. Agrawal, Fiber-Optic Communication Systems, 2nd ed. New York: Wiley, 1997.

[16] O. Leclerc, "Optical vs. electronic in-line signal processing in optical communication systems: An exciting challenge for optical devices," in Proc. Tech. Dig. Eur. Conf. Integrated Optics (ECIO), Prague, Czech Republic, 2003, pp. 55-67.

[17] B. Lavigne, D. Chiaroni, P. Guerber, L. Hamon, and A. Jourdan, "Improvement of regeneration capabilities in semiconductor optical amplifierbased 3R regenerator," in Proc. Optical Fiber Communication (OFC), San Diego, CA, 1999, p. 128.

[18] D. M. Baney, P. Gallion, and R. S. Tucker, "Theory and measurement techniques for the noise figure of optical amplifiers," Opt. Fiber Technol., vol. 6, no. 2, pp. 122-154, Apr. 2000.

Filip Öhman received the M.Sc. degree in engineering from the Royal Institute of Technology (KTH), Stockholm, Sweden, in 2001 and the Ph.D. degree from Research Center for Communications Optics and Materials (COM), Technical University of Denmark (DTU), Lyngby, in 2005.

His research interests are optical signal processing and noise in nonlinear semiconductor devices. In particular, he is investigating signal regeneration in semiconductor optical amplifiers (SOAs) and electroabsorbers through theoretical modeling and experimental characterization. He is currently an Assistant Professor at $\mathrm{COM}$

Jesper Mørk received the M.Sc., Ph.D., and Dr. Techn. degrees from the Technical University of Denmark (DTU), Lyngby, in 1986, 1988, and 2003, respectively.

Since 2002, he has been a Professor in semiconductor devices for opticalcommunication systems and is the Deputy Head of the nanophotonics area at Research Center COM. His current research interests are in the area of device physics, in particular ultrafast devices for optical signal processing, noise in nonlinear devices, and quantum photonics. 